\title{
Impulse corona simulation for flue gas treatment
}

\author{
Ivo Gallimberti \\ Department of Electrical Engineering, Padova University, Italy
}

\begin{abstract}
The lack of experimental results on transport coefficients in flue gases makes the macroscopic discharge models not applicable to the simulation of impulse corona for flue gas treatment. In the present paper the microscopic formulation, based on Boltzmann and Poisson's equations, is analyzed and a simplified model is derived. This has been numerically implemented in two sections: in the first one a quasi-uniform, steady-state Boltzmann equation is solved to calculate the electron and ion transport characteristics in any mixture of atmospheric gases $\left(\mathrm{N}_{2}, \mathrm{O}_{2}, \mathrm{CO}_{2}, \mathrm{H}_{2} \mathrm{O}\right)$; in the second section the conservation equations for electron, positive and negative ions are solved, together with Poisson's equation.

The model has been applied to the simulation of impulse corona discharges in air and flue gases with different compositions. The results indicate that $\mathrm{H}_{2} \mathrm{O}$ and $\mathrm{O}_{2}$ are the mixture components that play a major role in the attachment processes (at high and low field respectively); as a consequence the corona characteristics are very much affected by the flue gas composition. The optimization of any treatment process must therefore account for the possible variations of the gas composition; the admixture of gaseous products that lead to an improvement of pulse corona characteristics may also be considered.
\end{abstract}

\section{INTRODUCTION}

The growing concern about the quality of the environment has led many Research Laboratories and Electricity Boards to devote more and more attention to new technologies for the removal of polluting agents from industrial flue gases, namely the fly ash and the acid rain precursors $\mathrm{NO}_{x}$ and $\mathrm{SO}_{2}$.

The removal of $f$ ly ash is achieved by Electrostatic Precipitators, with the adoption of tall stacks for diluting the residual ashes in the atmosphere before they reach the ground level. However, the performances of conventional precipitators are limited by the high installation costs, so that problems may arise in countries with high population density, as the plant size and the number of plants in opertion are increased.

The removal of $\mathrm{NO}_{x}$ and $\mathrm{SO}_{2}$ may be obtained with Scrubbers, Catalityc Treatments or Electron Beam Irradiation: however, the development of these technologies is relatively recent, so that the industrial applications to large power plants are limited; in any case the estimated costs for installation and operation are very high.

The Italian program on "Flue Gas Clean-up" is mainly devoted to test and develop new techniques and operating procedures for reducing the pollutant effluent concentrations within the permissible limits proposed by the Commission of the European Communities. The research effort is mainly devoted to develop the following processes:

- the improvement of the electrostatic precipitator efficiency by means of impulse voltage application, preionization and conditioning of the flue gas (ref, 1,2); with these techniques a better charge deposition within the gap can be obtained, together with higher charging efficiency and migration velocity;

- the simultaneous $\mathrm{NO}_{x}$ and $\mathrm{SO}_{2}$ removal by means of impulse corona energization of the flue gas (ref. 3). With such a technique ions and oxidizing radicals 10 , $\mathrm{OH}, \mathrm{HO}_{2}$, etc.) are produced in the flue gas: the reactions of $\mathrm{NO}_{x}$ and $\mathrm{SO}_{2}$ with the radicals form several acids that can: be neutralized by injecting in the gas ammonia or lime; the final products can be removed from the flue gas in an electrostatic precipitator.

In both these applications, the knowledge of the pulse corona discharge is of great importance for the control and the optimization of the process efficiencies (ref. 4). However, the experimental and theoretical results on the discharge characteristics in flue gases are very poor so that generally a direct similarity with atmospheric air discharges is assumed. The few theoretical simulations have generally assumed the electron transport 
coefficients of normal air, neglecting the influence of relatively high concentrations of $\mathrm{H}_{2} \mathrm{O}$ and $\mathrm{CO}_{2}$. Therefore, the control of the high voltage energization and of the corona discharge is realized on the basis of very empirical criteria, so that the optimum conditions for the process efficiency are rarely realized.

The present paper reports the first results of an experimental and theoretical investigation on impulse corona characteristics in practical ESP gas, whose final objective is to define the optimum energization conditions in applications to particulate and oxides removal.

The computed swarm characteristics of the flue gas are discussed, and the expected differences between pulsed coronas in pure air and in flue gas are analyzed. A model of the impulse corona is then described and applied to positive streamers in both air and flue gas. The simulation results put in evidence the peculiarities of the ESP gas and indicate that in this case all assumptions of air corona similarity are far to be acceptable.

\section{GENERAL FORMULATION OF THE MODEL}

Under impulse conditions, the structure and the formation mechanism of the positive corona are very different from those under DC conditions (ref. 5). With DC voltages, the space charge alters the field distribution in the gap so that the ionization processes can occur in a diffuse form only within a narrow layer around the high voltage electrode. With impulse voltages, the ionization grows from the high voltage electrode into a background field which is purely geometric; the discharge assumes the from of a number of branched, tree-like streamers, whose starting points are discrete and distributed over the highly stressed region with a specific density.

Streamer discharges are highly localized positive or negative "space charge waves" (Fig. 11 , which enhance the applied field in front of the wave (active region) and propagate because of electrons avalaching in this high field (ref. 6). Photoionization is generally assumed to be the mechanism that supplies secondary seed electrons and triggers the avalanches. Along the track of the wave remains a weakly ionized plasma filament (passive region), along which the conduction current flows to the high voltage electrode, and supplies the energy for the streamer advancement.

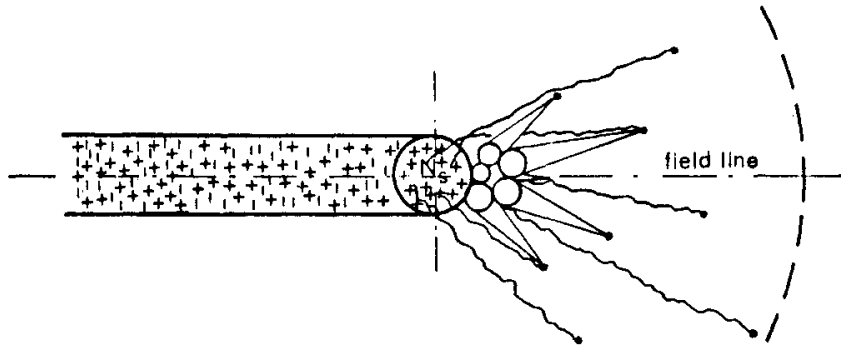

Fig. 1. Schematic representation of the propagation mechanism of positive corona streamers.

The high field created by the "space charge wave" makes possible its propagation also in relatively low applied field regions (much lower than the "critical field" that corresponds to the static breakdown field). Experimental and theoretical studies (ref. 7,8 ) have made possible to define a "stability field", which is the externally applied field which makes the propagation of positive streamers energetically stable: in air it results around 5 $\mathrm{kV} / \mathrm{cm}$.bar. In higher externally applied fields, the streamer space charge wave increases in size and stores potential energy; in lower applied fields the propagation dissipates the stored poptential energy until the propagations stops.

Recent experimental and theoretical studies (ref. 9,10 ) have shown that the propagation characteristics of positive streamers depends very much on the degree of electronegativity of the buffer gas. In weakly electronegative gases (like atmospheric air) the streamers appear much longer and more branched that in strongly electronegative gases (like $\mathrm{SF}_{6}$ ): the corresponding currents and charges are also much higher, and the average field along the streamer track increases from the "stability value" to the "critical value". The transition between the weakly-electronegative behaviour to the strongly-electronegative behaviour has been shown to occur when the attachment mean free path becomes of the same order of magnitude as the size of the streamer head. The degree of electronegativity of the flue gas is just across the transition region: with high $\mathrm{H}_{2} \mathrm{O}$ and $\mathrm{CO}_{2}$ concentrations the discharge characteristics are very near to those of strongly electronegative gases, while with low contents of $\mathrm{H}_{2} \mathrm{O}$ and $\mathrm{CO}_{2}$ they are quite similar to those observed in normal air. 
parameters (type of fuel, characteristics of burners, boiler load, air flow, etc.) which are variable in time and from plant to plant. In average, the basic components of the ESP gas, whose concentration can affect the transport characteristics of an electron swarm, are reported in Table 1: the main difference with respect to atmospheric air is the presence of $\mathrm{H}_{2} \mathrm{O}$ and $\mathrm{CO}_{2}$ at the place of $\mathrm{O}_{2}$; the percentage of $\mathrm{H}_{2} \mathrm{O}$, which is generally higher for oil than for coal fuels, is responsible for the major changes in the swarm characteristics, because of the high value of the attachment cross section.

TABLE 1. Average composition of the exhausted gases of a power plant depending on the type of fuel.

\begin{tabular}{|c|c|c|c|c|c|c|c|}
\hline FUEL & $\mathrm{N}_{2}$ & $\mathrm{O}_{2}$ & $\mathrm{H}_{2} \mathrm{O}$ & $\mathrm{CO}_{2}$ & $\mathrm{CO}$ & $\mathrm{SO}_{2}$ & NO \\
\cline { 2 - 3 } OIL & $72 \%$ & $1 \%$ & $12 \%$ & $15 \%$ & $100 \mathrm{ppm}$ & $1500 \mathrm{ppm}$ & $300 \mathrm{ppm}$ \\
COAL & $74 \%$ & $5 \%$ & $6 \frac{15}{3}$ & $15 \%$ & $40 \mathrm{ppm}$ & $700 \mathrm{ppm}$ & $600 \mathrm{ppm}$ \\
\hline
\end{tabular}

No experimental results are available in the literature on electron and ion transport coefficients in flue gases, which could characterize the macroscopic behaviour of a swarm of charged particles under the action of an electric field. No macroscopic models $($ ref. $6,8,10)$ can be therefore applied to the simulation of corona discharges in flue gases. Microscopic models based on the kinetic of elementary collisions have to be developed (ref. $11,12,13)$.

Let's consider an assembly of identical charged particles of type "i" (electrons or ions), of mass $m_{x}$ and charge $q_{1}$, in a weakly ionized gas: the state of this assembly can be described statistically at a microscopic level by the distribution function $\mathbf{E}_{1}(\underline{\boldsymbol{r}}, \underline{\mathbf{v}}, t)$ in the position and velocity space $(\underline{\mathbf{r}}, \underline{\mathbf{v}})$.

The particle density at any position $\underline{r}$ and time $t$ can be obtained from this function by integrating over the velocity distribution

$$
n_{1}(\underline{r}, t)=\int f_{1}(r, \underline{v}, t) d \underline{\underline{v}}
$$

The function $f_{1}$ obeys the basic conservation equation (Boltzmann Equation),

$$
\frac{\partial \mathrm{f}_{1}}{\partial \mathrm{t}}+\underline{\mathrm{v}} \cdot \nabla_{\mathrm{r}} \mathrm{E}_{\mathrm{i}}+\frac{\mathrm{q}_{1}}{\mathrm{~m}_{1}} \underline{\mathrm{E}} \cdot \nabla_{\mathrm{v}} \mathrm{f}_{1}=I\left(\mathrm{f}_{1}\right)
$$

where $\underline{E}(\underline{r}, t)$ represents the actual electric field (due to the externally applied voltage and to the space-charge distribution), and $I\left(f_{1}\right)$ is a non linear "source term", which accounts for the change of particle position and velocity in the $(\underline{r}, \underline{v})$ space, because of collisions with neutral molecules and other charged particles.

The electric field is given by the Poisson's Equation

$$
\nabla_{x} \cdot \underline{E}(\underline{\underline{r}}, t)=\sum_{1} \frac{q_{1} n_{1}(\underline{\underline{r}}, t)}{\varepsilon}
$$

where the sum extends over all the charged species, and $\varepsilon$ represents the dielectric constant of the background medium.

In the plasma of corona discharges the density of charged particles is so low with respect to neutrals that the following simplifying assumptions are generally acceptable:

- the collisions between charged particles and neutral molecules are binary, instantaneous and punctual in space;

- the interactions between charged particles can be neglected, as their fxequency is so low that the distribution functions $f_{1}$ are not disturbed;

- the distribution function of neutral molecules is maxwellian (at the gas temperature $T$ ) and not affected by momentum and energy transfer from charged particles.

Under these conditions the operator $I\left(f_{1}\right)$ becomes linear (ref. 11); it results from the 
balance of two terms, representing respectively the population and depopulation rates of the control volume around position $\underline{r}$ and velocity $\underline{v}$, because of collisions between particles of type " $i$ " and neutral molecules (ref, 12):

$$
I\left(f_{1}\right)=f_{1}(\underline{\underline{\underline{v}}}, \underline{\mathbf{v}}, t) \cdot\left[-\underline{\underline{v}} \sum_{k} N_{k} \sigma_{1 k}(\underline{\underline{v}})+\sum_{k} N_{k} \nu_{i k}\left(\underline{\underline{v}}, \sigma_{i k}, f_{1}\right)\right]
$$

where $v$ represents the absolute value of $\underline{v}, \sigma_{1 x}$ the cross section for collisions of type "k", $\mathrm{N}_{x}$ the density of the neutral species responsible for collisions of type " $k$ " and $\boldsymbol{\nu}_{i k}\left|v, \sigma_{i k}, \tilde{E}_{1}\right|$ the rate of population of the velocity level $\underline{\mathbf{v}}$, due to deceleration of faster particles in collisions of type " $k "$.

Because of the complexity of the collision operator $I\left(f_{1}\right)$ and the number of variables, a general solution of the Boltzmann and Poisson's equations at the microscopic level is in practice impossible, either analitically or numerically.

As the time-scale of interest in the macroscopic simulation of corona discharges is much longer than the time-scale of individual collisions, the general Boltzmann equation may be replaced (ref. 13) by a group of "moment equations" : each of these can be obtained by taking appropriately weighted integrals in $\mathbf{v}$ space of the Boltzmann equation; in this case the "kinetic model" is replaced by an "hydrodynamic model". The first three moment equations (namely density $n_{1}(\underline{r}, t)$, average velocity $\underline{w}_{1}(\underline{r}, t)$ and mean kinetic energy $\varepsilon_{1}(\underline{r}, t)$ are

$$
\begin{aligned}
& \frac{\partial n_{1}}{\partial t}+\nabla_{x} \cdot\left(n_{1} \underline{w}_{1}\right)=\nu_{1} n_{1} \\
& \frac{\partial\left(n_{1} \underline{w}_{1}\right)}{\partial t}+\nabla_{x}\left\langle n_{1} \underline{v} \cdot \underline{v}\right\rangle-\frac{q_{1}}{m_{1}} \underline{E} \cdot n_{1}=-\nu_{1 m} n_{1} \underline{w}_{1} \\
& \frac{\partial\left(n_{1} \varepsilon_{1}\right)}{\partial t}+\nabla_{r}\left\langle n_{1} \varepsilon_{i} \underline{v}\right\rangle-q_{1} E n_{1} \underline{w}_{1}=-\nu_{i \varepsilon} n_{1} \varepsilon_{1}
\end{aligned}
$$

where the brackets imply average over the velocity distribution, and ${ }^{\prime}$, tm' io are respectively the effective production/loss frequency of " $i$ " particles (by ionization, attachment or other processes), the momentum-exchange collision frequency and the energyexchange collision frequency: they are defined by

$$
\begin{aligned}
& \underline{\nu}_{1}=\frac{1}{n_{1}} \int I\left|f_{1}\right| d \underline{v} \\
& \underline{w}_{1} \nu_{1 m}=-\frac{1}{n_{1}} \int \underline{v} \cdot I\left(E_{1} \mid d \underline{v}\right. \\
& \varepsilon_{1} \nu_{1=}=-\frac{1}{n_{1}} \int \frac{1}{2} m_{1} v^{2} \cdot I\left(f_{1}\right) d \underline{v}
\end{aligned}
$$

The set of equations (5) can be further simplified on the basis of time-scale considerations. In a discharge plasma, the energy-exchange and momentum-exchange collision frequencies are generally much larger than the charge carrier production frequency. This means that the time constants $\tau_{1 m}=1 / \nu_{1 m}$ and $\tau_{1=}=1 / \nu_{1}$ for reaching a local equilibrium of $\underline{w}_{1}$ and $\varepsilon_{1}$ with the local field $\underline{E}$ are much shorter than the time constant $\tau_{1}=1 / \nu_{1}$ for charge carrier production. As the latter fixes the time-scale of ionization groth in the simulation of corona discharges, the time and position derivatives in the second and third of equations (5) can be neglected; these two equations become therefore simple algebraic equations that make possibile, in conjunction with eqs. $(6)$, to calculate the average velocity $\underline{w}_{2}$ and the mean energy $\varepsilon_{1}$.

Unfortunately the set of equations $(3),(5)$ and $(6)$ is not determined: in fact equations (5) contain unknown frequencies; to calculate them, the complete distribution function $f_{1}$ is needed, which implies having to solve the original Boltzmann equation. 
This can be overcome with a simplified approach to the distribution function: when the particle density $n_{1}(\underline{r}, t)$ varies only slightly over the characteristic distances of individual collisions, the deviation from spatial uniformity is small and a perturbation expansion can be used for the distribution function $f_{1}(\underline{\underline{r}}, \underline{v}, t)$ (ref. 11 ). In the zero-th order term of this expansion the variables can be separated, and it results

$$
f_{1}(\underline{\underline{r}}, \underline{v}, t)=\mathbf{f}_{1}^{0}(\underline{\underline{v}}) \cdot n_{1}(\underline{r}, t)
$$

where $f_{1}^{0}(\underline{v})$ obeys the uniform, steady state Boltzmann equation

$$
\frac{q_{1}}{m_{1}} E \cdot \nabla_{v} E_{1}^{0}=I\left(E_{2}^{0}\right)-E_{1}^{0} \int I\left(E_{1}^{0}\right) d \underline{v}
$$

with the normalization condition

$$
\int \mathbf{f}_{1}^{0}(\underline{\mathbf{v}}) \mathbf{d \underline { \mathbf { v } }}=1
$$

If the solution of equation $(8)$ is numerically calculated, than it can be used in conjunction with equations $(7)$ and $(4)$, to calculate the unknown frequencies in equations $(6)$.

The simplyfing assumption of eq. 171 implies physically that the distribution function $\mathfrak{E}_{1}$ is locally not dependent on the particle density (because it is assumed to vary not too rapidlyl, so that the charged particles can assume a velocity distribution $f_{1}^{\circ}$ in quasiequilibrium with the local field E. In fact the solution $f_{1}^{0}$ of eq. ( 8 ), for given gas characteristics /component densities $N_{k}$ and cross sections $\left.\sigma_{1 k}\right)$, depends only on the local field E. As a consequence the collision operator in eq. (4) becomes proportional to the density $n_{1}$, and the collision frequencies in eqs. (6) become functions only of the local field $\underline{E}$.

The simulation model for corona discharges in flue gases is made up of two sections: in the first one the uniform, stready-state Boltzmann equation (8), is solved for different values of the field, according to the gas composition and to the collisional cross-sections of the individual components; then the collision frequencies are calculated together with the drift velocities and mean energies (eqs. 6 and 5). In the second section the set of moment equations $(5)$ for electrons, positive and negative ions are solved together with Poisson's equations (3), for the specific conditions of pulse corona streamers.

\section{SOLUTION OF THE BOLTZMANN EQUATION}

The time scale for pulse corona formation, in practical ESP field configurations, is typically of the order of $10^{-7} \mathrm{sec}$. In this time the positive and negative ions can not move more than $10^{-3} \mathrm{~cm}$; as this length is negligeable with respect to the electrode distance, the velocities $w_{+}$and $w_{-}$of positive and negative ions can be assumed zero: therefore, it is not necessary to solve the Boltzmann equation for ions; they are only included as still particles in the set of hydrodynamic equations.

The Boltzmann equation (8) has been solved numerically for electrons, with reference to the electron-molecule collision cross sections. The basic components of the flue gas are listed in Table 1: however, the components whose density is only of some 100 ppm do not affect significantly the distribution function $\mathrm{f}_{1}^{\circ}(\mathbf{v})$. Therefore, collisions with these components have been neglected. in the collision term (equation 4), and only the cross sections of the basic components $\left(\mathrm{N}_{2}, \mathrm{O}_{2}, \mathrm{H}_{2} \mathrm{O}, \mathrm{CO}_{2}\right)$ have been taken into account.

Experimental values of most of the relevant cross sections are available in the literature (ref. 14,15); however, the values are in many cases contradictory, the absolute calibration beeing in general difficult and strongly dependent on the experimental technique.

In order to obtain a reasonable set of cross sections for each component, the literature values have been fitted and then tentatively optimized (ref. 16). This optimization has been realized by solving numerically the Boltzmann equation in the parent gas (pure nitrogen, oxygen, etc.l and by comparing the computed transport characteristics laverage velocity, mean energy and collision frequencies/ with the experimental results which are available in the literature for pure gases: the cross sections have been then adjusted to obtain the best agreement between computed and experimental transport parameters, over a very wide range of applied fields. 
For nitrogen and oxygen the cross-section sets obtained by the JILA Group (ref. 17) have been used, with only minor modifications; for carbon dioxide and water waper the optimized cross section sets have been derived in a collaboration with the CPAT Group (ref. is). In these sets about one hundred electron molecule collision processes are taken into account. In order to check the validity of the cross-section sets in gas mixtures, the transport parameters have been also calculated in dry and humid air and compared with the available experimental results. The comparison is quite satisfactory, indicating that the actual sets of cross-sections are reasonably consistent (ref. 19).

These data have been assumed as the initial core of a Data-Base on Cross-sections and Transport-parameters by the European Group on Gas Discharges: further work of selection and optimization is going on within this Group to obtain more refined and reliable data.

The numerical technique for the solution of the Boltzmann equation has been developed by the CPAT Group (ref. 18): it is based on a finite element discretization algoritm, which ensures very high accuracy of the results, together with a reasonably fast and stable convergence.

In the present paper three sample cases have been treated: the dry air and the two standard flue gases reported in Table 1 , for oil and coal feul respectively lin the following they will be referred as ESP I and ESP II mixtures).

The electron distribution function $\mathrm{f}^{\circ}(\mathbf{y})$ has been calculated for the different mixtures at different values of the reduced applied field $\mathrm{E} / \mathrm{N}$ : a few examples are reported in Fig. 2 . The high energy tail of the distribution function increases as $E / N$ is increased, but remains almost the same for all the three mixtures: it is mainly determined by the $\mathrm{N}_{2}$ buffer gas, which absorbs 85-90\% of the energy transferred from the electrons to the gas, and therefore defines the spectrim of energy losses.

As a consequence the electron drift velocity $w_{a}$ and mean energy $\varepsilon$. are not significantly affected by the ESP gas composition (Figs. 3a) and $3 b$ ). The same occurs for the energyexchange and momentum-exchange collision frequencies $\nu_{1}$. and $\nu_{1 \mathrm{~m}}$.

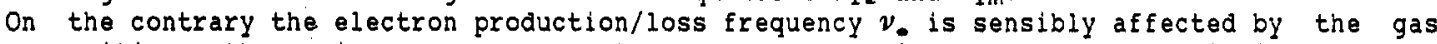
composition: the main processes that have been taken into account are ionization and attachment lelectron-ion recombination has a low probability because of the low densities, and detachment of negative ions my be included in the net attachment ratel; the frequency $\nu$. may be therefore expressed as

$$
\nu_{0}=\alpha w_{a}-\eta w_{a}
$$

where $a$ and $\eta$ are the lonization and attachment coefficients. The computed values of $\alpha$ and $\eta$ are reported in Figs. $3 \mathrm{c}$ ) and $3 \mathrm{~d}$ ): the ionization rate is not significantly affected by the gas mixture. On the contrary the attachment coefficient is much larger in ESP II at high field, and in air at low field. The main attachment process at high field is the dissociative attachment of $\mathrm{O}_{2}, \mathrm{H}_{2} \mathrm{O}$ and $\mathrm{CO}_{2}$; the higher rate is that of $\mathrm{H}_{2} \mathrm{O}$, whose concentration is therefore the dominant parameter for defining the value of the attachment coefficient.

At low field, when the average electron energy becomes much lower than the disscciation thresholds, the dominant attachment process is the oxygen three-body attachment. In this case the oxygen concentration is the dominant parameter for defining the value of the attachment coeficient.

The above presented results make possible to calculate in the different mixtures the static breakdown as the critical field value at which the ionization and attachment rates become equal (Fig. 4): it is mainly dependent on the $\mathrm{H}_{2} \mathrm{O}$ concentration, and shows a variation of about 20-30 according to the flue gas composition.

\section{SOLUTION OF THE HYDRODYNAMIC SYSTEM}

The model for the hydrodynamic regime of the charge carriers, can be derived from eqs. (5) and $(6)$, with the proper simplifications, due to the short time-scale of the energy-exchange and momentum-exchange processes, and to the long time-scale of the ion motion:

$$
\begin{aligned}
& \frac{\partial \mathrm{n}_{\bullet}}{\partial \mathrm{t}}+\nabla_{x} \cdot\left(\mathrm{n}_{-} \underline{w}_{-}\right)=v_{\bullet} \mathrm{n}_{-} \\
& \frac{\partial \mathrm{n}_{+}}{\partial \mathrm{t}}=v_{+} \mathrm{n}_{+} \\
& \frac{\partial \mathrm{n}_{-}}{\partial \mathrm{t}}=v_{-} \mathrm{n}_{-} \\
& \nabla_{x} \cdot \underline{\underline{E}}=\frac{\left(n_{-}+n_{-}-n_{+}\right) \cdot q_{-}}{\varepsilon}
\end{aligned}
$$

where $n_{-}, n_{+}$and $n_{-}$represent the electron, positive and negative ion densities and $q_{0}$ the electron charge. 


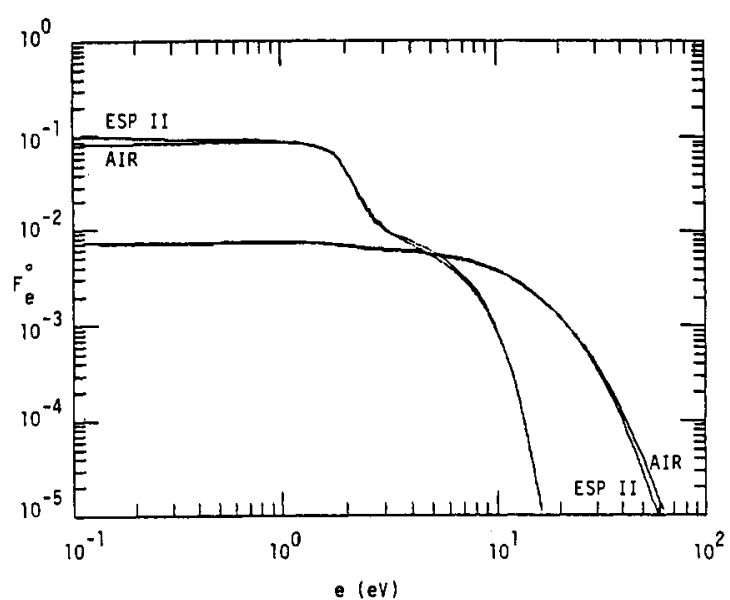

Fig, 2, Computed electron energy distributions for air and flue gas (ESP II), at different values of the reduced field $E / N$.
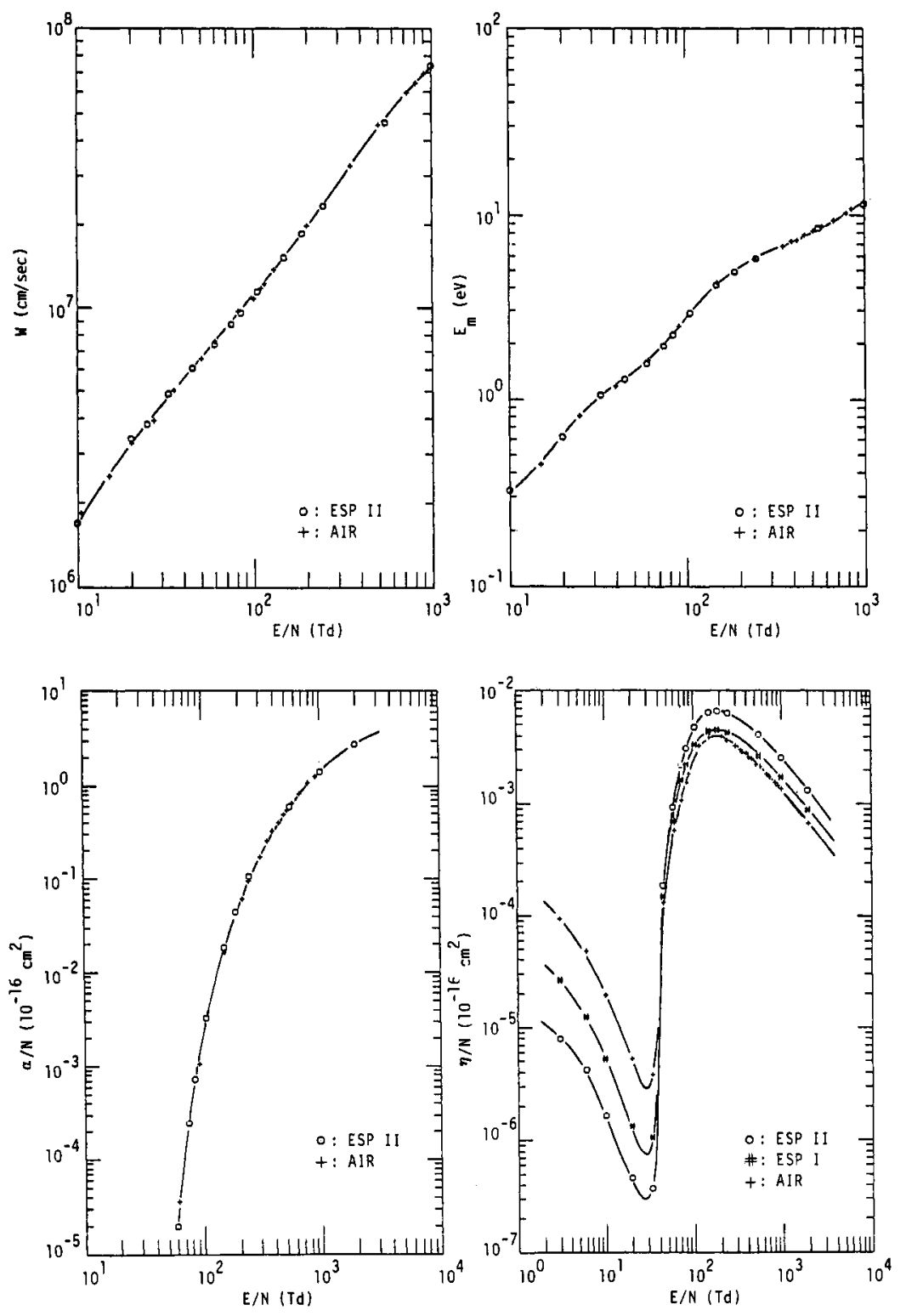

Fig. 3. Computed electron transport parameters in air and flue gas, as functions of the reduced field $E / N$, al Drift velocity $W_{a}$ i bl mean energy $\varepsilon_{0} ;$ c) ionization coefficient $a / N$; d) attachment coefficient $\eta / N$. 
Some more discussion is needed for the electron average velocity $\underline{w}_{0}$ and for the production frequencies $\nu_{-}, \nu_{+}$and $\nu_{-}$.

The average electron velocity computed from the solution of the Boltzmann equation $(8)$ is the pure drift velocity due to the electric field; in fact the assumed independence on the electron density (spatial quasi-uniformity) implies that no diffusion components are included. However, in the ionization front of a streamer channel large electron density gradients may be present, so that diffusion has to be included as a component of the average electron velocity

$$
\underline{w_{*}}=\underline{w_{d}}-\mathrm{D} \nabla_{x} \cdot \mathrm{n}_{\boldsymbol{*}}
$$

The diffusion coefficient $D$ can be derived from the electron drift velocity and mean energy

$$
D=\frac{w_{0}}{E} \cdot \frac{2}{3} \varepsilon \text {. }
$$

The electron production frequency given by equation $(10)$ is in general not enough for the simulation of corona streamers. It has been in fact demonstrated (ref. 6$)$ that their propagation needs a secondary ionization mechanism for producing the seed electrons that sustain the avalanche formation in the ionization front. This secondary process is generally associated to photoionization: it has a frequency much smaller than the values of $\nu_{e}$ given by eq. 10 , so that it is correct to neglect it in the solution of the Boltzmann equation; on the contrary it has to be included in the "source term" of the conservation equations (11) because, even if small, it is necessary for the self-sustained propagation of streamer channels.

The photoionization term can be estimated at position $\underline{r}$ as

$$
\nu_{p h}(\underline{\underline{r}}, t)=\sum_{k} \eta_{k} u_{k} \delta_{k} \cdot \frac{\left.n_{0}\left(\underline{r}_{0}, t\right) w_{d} \exp \left|-\mu_{k}\right| \underline{r}-\underline{r}_{0} \mid\right)}{4 \pi\left|r-r_{0}\right|^{2}} d \underline{r}_{0}
$$

where the sum is extended over all the transitions of energy higher than the ionization thereshold; $\delta_{k}$ represents the excitation coefficient of the upper state, $\mu_{k}$ and $\eta_{k}$ the absorption coefficient and the photoionization efficiency, and $\underline{r}_{0}$ the variable position where photons are emitted.

In the ion production terms on the contraxy the photoionization contribution can be neglected; they can be therefore simply expressed in the form

$$
\nu_{+} n_{+}=a n_{0} w_{0} ; v_{--} n_{-}=n_{*} w_{a}
$$

In the actual model the streamer filament is assumed to have a rotational simmetry with a constant radius $R$, equal to the diffusion radius of an avalanche producing the critical charge of $10^{8}$ electrons (around 30-35 um); all the particle densities on a transversal section are assumed to have a gaussian profile, which in fact derives from the radial diffusion of the avalanche head (ref. 20). Radial diffusion of the streamer channel. is neglected because it occurs essentially at ambipolar rate.

The set of hydrodynamic equations 1111 can be therefore rewritten in the form:

$$
\begin{aligned}
& \frac{\partial n_{\bullet}}{\partial t}+\frac{\partial\left(n_{-} w_{a}\right)}{\partial x}-D \frac{\partial^{2} n_{\bullet}}{\partial x^{2}}=(\alpha-\eta) n_{a} w_{a}+\nu_{p h} \\
& \frac{\partial n_{+}}{\partial t}=a n_{-} w_{a} \\
& \frac{\partial n_{-}}{\partial t}=\eta n_{a} w_{a} \\
& \nu_{p n}=\sum_{k} \eta_{x} H_{k} \delta_{k} \cdot \frac{2 n_{-} w_{a} R^{2} \exp \left(-H_{i} x\right)}{(2 x-R)^{2}} d x
\end{aligned}
$$

The electron and ion densities in this case represent the values they assume on the streamer axis. 
The Poisson's equation (3) is solved in its integral form, by considering the streamer filament divided in to a number of thin disks over which the net space charge is distributed with a gaussian profile. An analytical espression is derived to calculate the field of these thin disks, all along the discharge axis, and then the effect is cumulated numerically for all the disks (ref. 20 ).

The set of hydrodynamic equation $(16)$ is solved with a flux-corrected finite difference technique, in a step by step procedure in the time direction (ref. 21). The flux-correction in the finite difference expression of the spatial derivatives ensure a very good numerical stability (ref. 22 ).

Typical examples of the calculated results in air and in ESP II are reported in $F$ igs. 5. In Figs, 5a) and b) the advancing front of electron density is reported: the ionization growth in the active region is very rapid; then the electron density decreases in the streamer filament due to attachment. In the streamer track, a trail of positive and negative ions is created (Figs. $5 \mathrm{c}$ ) and d).

In flue gas the peak electron density in the advancing ionization front is smaller than in air, because of the larger value of the high-field attachment rate. On the contrary the decay tail of the electron density within the streamer filament is much longer because of the smaller value of the low-field attachment rate: correspondingly, in the flue gas the negative ion density" is build up within the streamer filament at a much slower rate.

These peculiar attachment characteristics are reflected in the calculated current pulse (Fig. 6): in the flue gas it has a lower peak value, but contains a larger charge.

The corona extention is smaller in flue gas (at the same applied voltage), and the "guiding field", defined as the average voltage drop necessary to propagate a streamer channel along a unit length, is correspondingly higher. In Table 2 the basic streamer characteristics in air and flue gas are summarized, for an applied voltage of $50 \mathrm{kV}$.
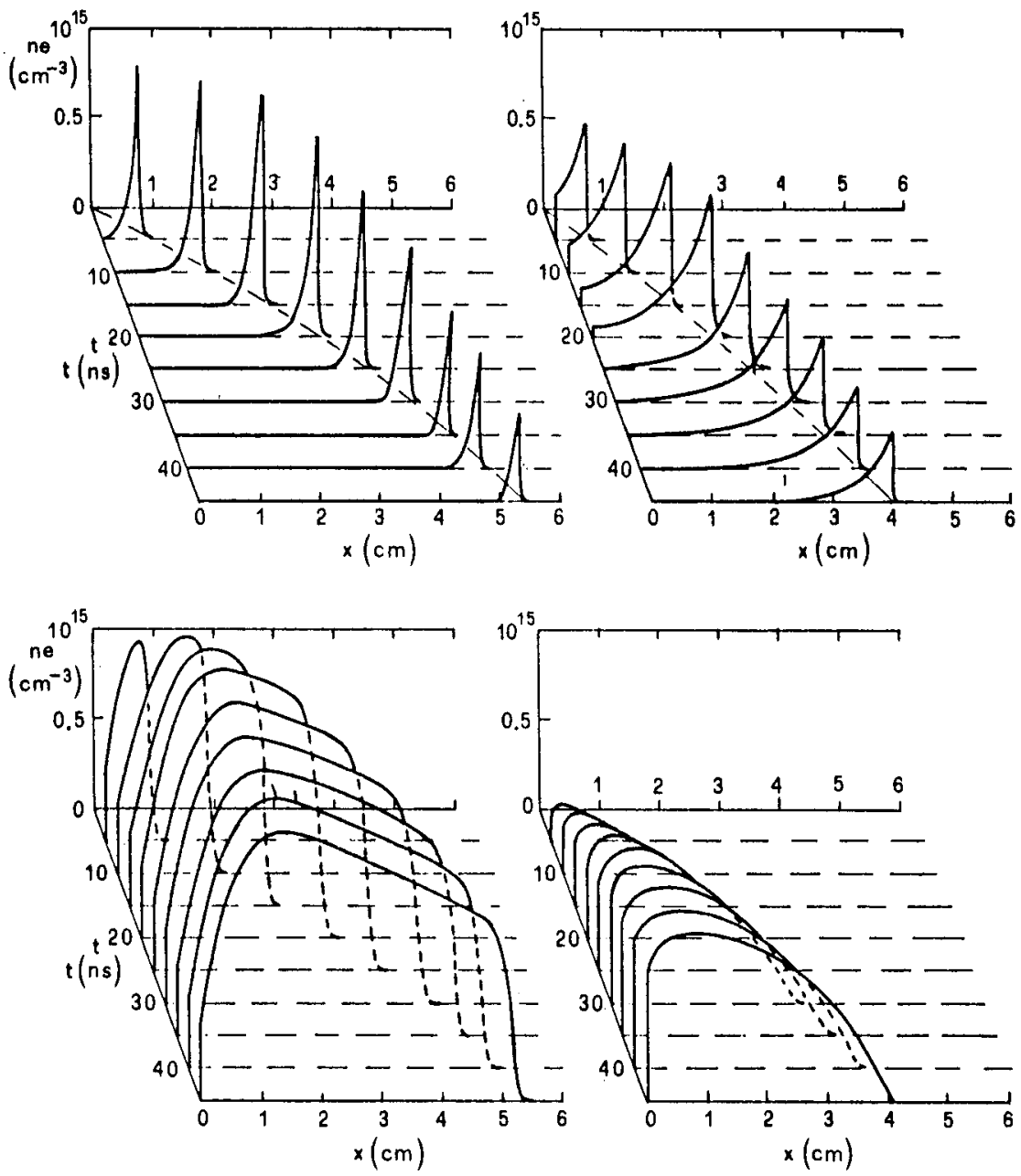

Fig. 5. Evolution in space and time of a positive streamer "space charge wave"; $10 \mathrm{~cm}$ wire-plane gap; $1 \mathrm{~mm}$ wire radius; $60 \mathrm{kV}$ applied voltage. al, b) electron density in air and ESP II respectively; cl, d) negative ion density in air and ESP II respectively. 

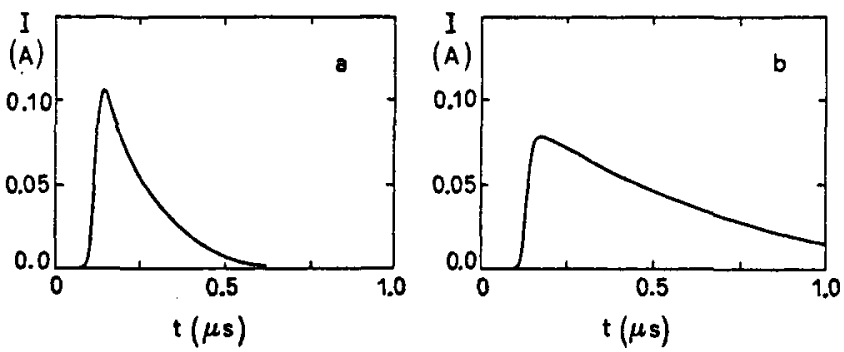

Fig. 6. Computed current pulses for the streamer simulations of Fig. 5.

a) air; b) flue gas (ESP II).

TABLE 2. Characteristics of a single corona streamer in air and flue gas; $10 \mathrm{~cm}$ wire-plane gap, $1 \mathrm{~mm}$ wire radius; $50 \mathrm{kV}$ applied voltage.

\begin{tabular}{|ll|r|r|r|}
\hline \multicolumn{1}{|c|}{ G A S } & AIR & ESP I & ESP II \\
\hline Guiding field & $(\mathrm{kV} / \mathrm{cm})$ & 4.5 & 4.3 & 8.1 \\
Streamer length & $(\mathrm{cm})$ & 10.0 & 10.0 & 4.2 \\
Net charge & $(\mathrm{nC})$ & 6.6 & 13.2 & 20.1 \\
Total Energy & $(\mathrm{HJ})$ & 414. & 723. & 563. \\
Charge formation Energy (HJ) & 4.7 & 4.3 & 2.4 \\
Charge separation Energy (uJ) & 409. & 718. & 560. \\
\hline
\end{tabular}

The variations of net charge and total energy with the applied voltage are presented in Fig. 7. The total energy associated with the streamer formation is spent partly in the ionizing front for the formation of the charge lenergy losses of the electrons avalanching in the high field region) and partly for the separation of the charge (energy losses of the electrons drifting in the low field channel until they remain attached). From Table 2 and Fig. 10 it can be seen that the first component decreases when the $\mathrm{H}_{2} \mathrm{O}$ concentration is increased because of its high rate of dissociative attachment, while the second component decreases when the $\mathrm{O}_{2}$ concentration is increased because of its high rate of three-body attachment. As the second component is the major one, the net charge and the total energy are higher in flue gas.

\section{CALCULATION OF THE RADICAL DENSITIES}

In order to simulate the chemical kinetic of a corona induced DeNO $\mathrm{D}_{x}$ and DeSO $\mathrm{x}_{\mathrm{x}}$ process (ref. 23) it is necessary to know the concentrations of the active radicals within the discharge volume.

By using the same technique described for the calculation of the electron transport characteristics, the dissociation coefficients have been computed for the different molecules in the flue gas mixture (Fig. 8). At relatively low fields the $\mathrm{CO}_{2}$ and $\mathrm{H}_{2} \mathrm{O}$ dissociation processes are the dominant one, because of the low energy threshold; at high fields the $\mathrm{N}_{2}$ dissociation becomes more probable because of the large concentration; the $\mathrm{O}_{2}$ dissociation rate is relatively small because of its low concentration. However, the dissociation coefficients of Fig. 8 may be affected by large errors, because of the large incertitudes in the dissociation cross-sections reported in the literature.

By using the dissociation coefficients, rate equations for radical production have been introduced in the streamer simulation model, to calculate the densities of radicals left in the gap by the corona formation. In Fig. I the computed results along a single streamer filament are reported for the ESP II flue gas. The dominant radical species are $H$ and $O H$, but a relatively high concentration of $O$ is also present, due mainly to the dissociation of $\mathrm{CO}_{2}$. The ionic species have a much lower density.

The efficiency of radical production is given in Fig. 10 by the $G$ parameter, which represents the number of radicals produced per $100 \mathrm{eV}$ of injected energy: it depends very much on the applied voltage and on the flue gas composition. If the voltage is raised the efficiency $G$ increases up to the voltage value necessary for the corona streamers to cross the gap; at higher voltages the efficiency decreases again. The $G$ values appear to be quite low, if compared with the typical values obtained with E-beam irradiation. This is due to the fact that the electrons deposit most of their energy (see Table 2 ) in the low fleld region of the streamer filament: here they have an average energy of the order of 1 eV, and cause almost only vibrational excitation. However it has to be stressed that all seconday processes are negleated in the present model las cascade processes, vibrational resonant pumping up to dissociation threshold, ecc.l: some of them can have efflciencies in radical production at low energy similar or even larger than the primary electron collisional dissociation.

The results presented in Fig. 10 are worth of some further discussion:

- the fractional composition of the radicals is not significantly affected by the voltage (Fig, 10a);

- the energy efficiency $G$ tends to increase if the content of both $\mathrm{O}_{2}$ and $\mathrm{H}_{2} \mathrm{O}$ is increased (Fig. 10b): 

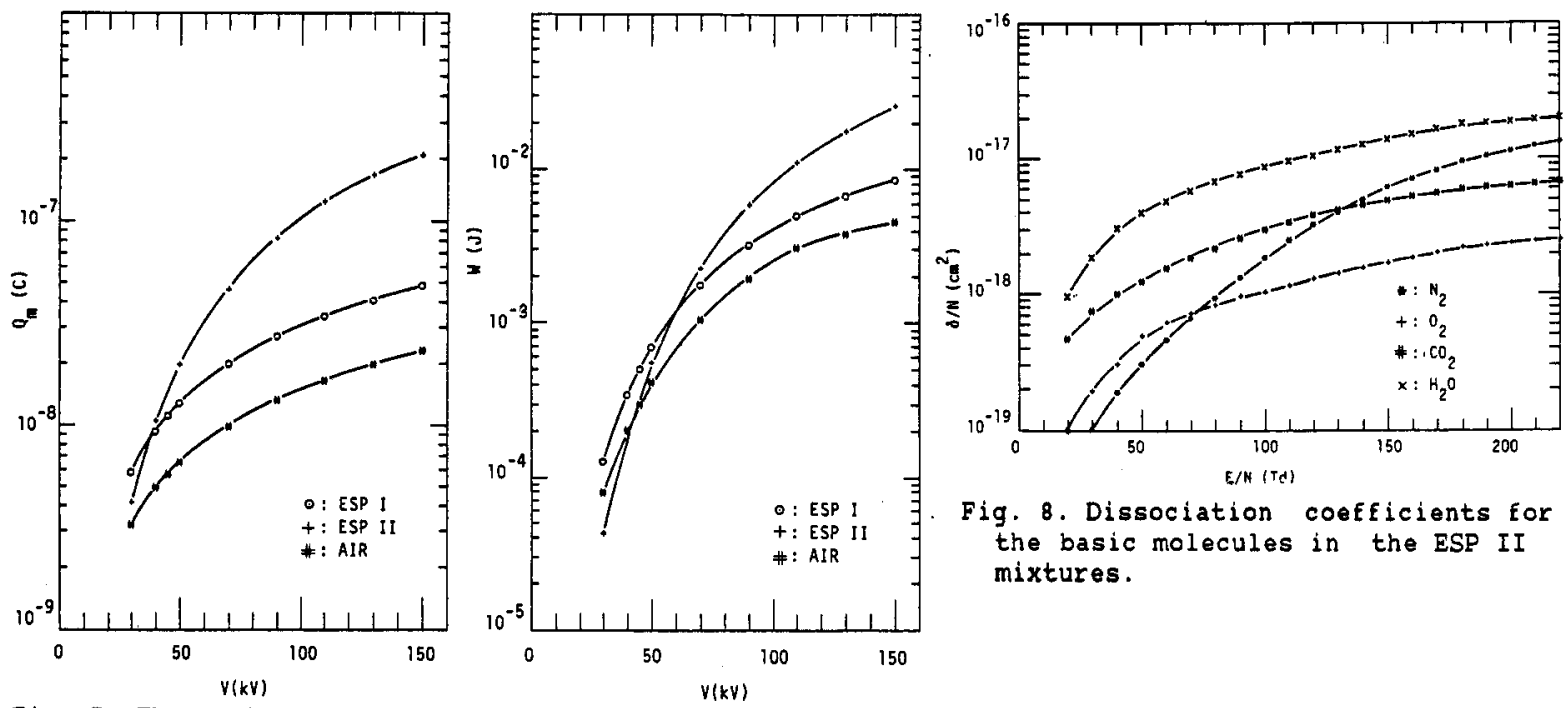

Fig. 8. Dissociation coefficients for the basic molecules in the ESP II mixtures.

Fig. 7. The net charge and total energy of a single streamer in air and flue gas as functions of the applied voltage; $10 \mathrm{~cm}$ wire-plane gap; $1 \mathrm{~mm}$ wire radius.
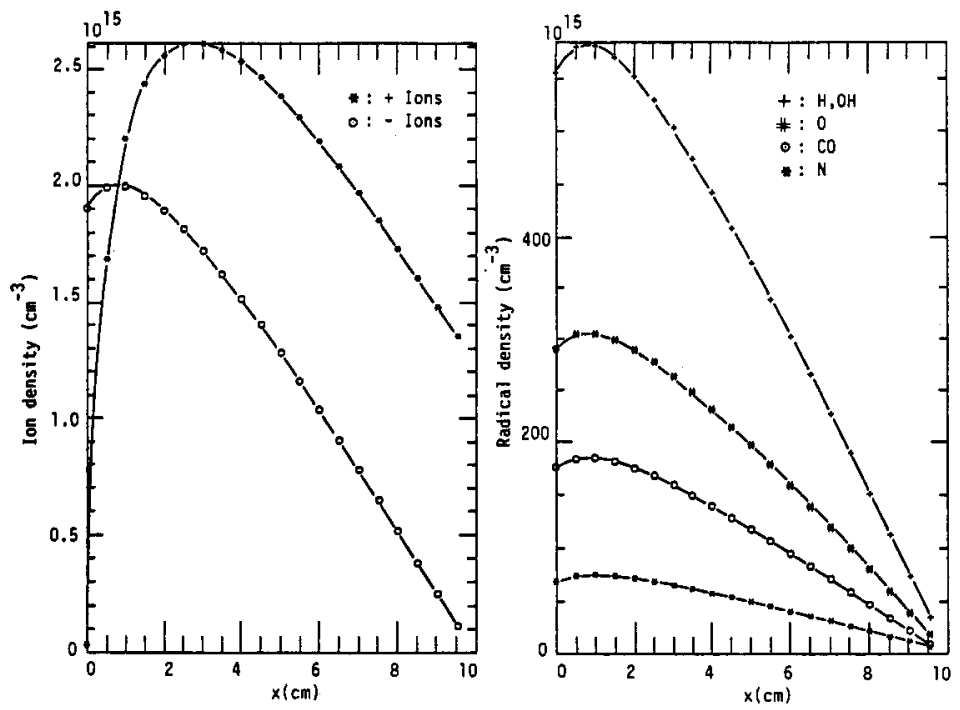

Fig. 9. Distribution of ions (a) and radicals (b) along a streamer channel; $10 \mathrm{~cm}$ wire-plane gap; $1 \mathrm{~mm}$ wire radius; $100 \mathrm{kV}$ applied voltage.
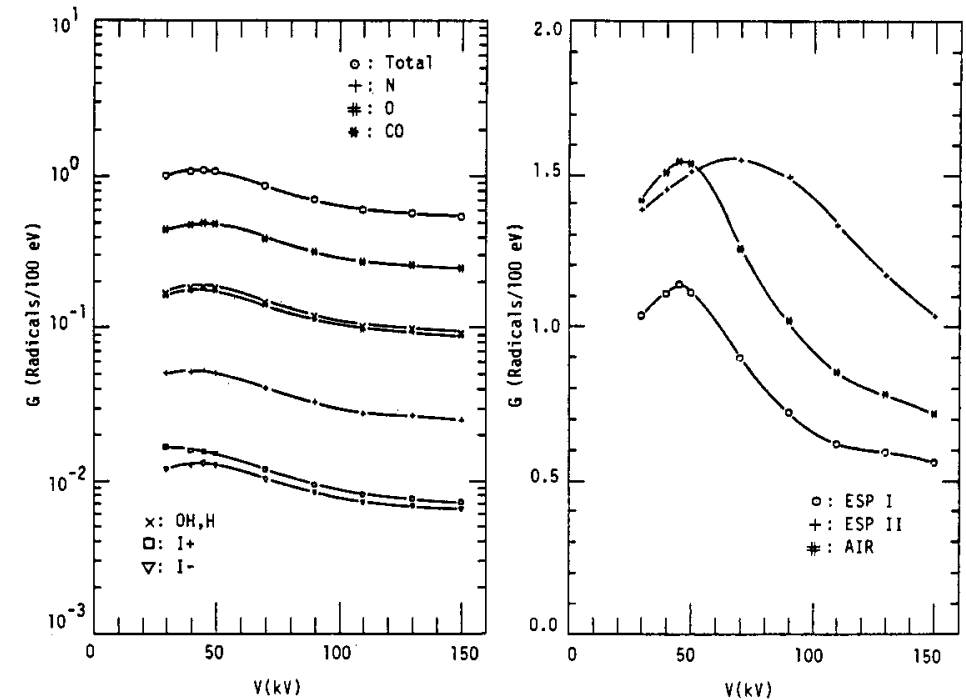

Fig. 10. The computed energy efficiency $G$ for radical formation by corona energization of flue gas as a function of the applied voltage; $10 \mathrm{~cm}$ wire-plane gap; $1 \mathrm{~mm}$ wire radius: a) the $G$ factor for individual species in ESP $I$; $b)$ the total $G$ factor in different mixtures. 
- the $\mathrm{O}_{2}$ effect is associated to the reduction of the mean attachment length in the low field streamer channel; this reduces the charge separation energy with respect to the charge formation energy; however, the oxygen reduces in the mean time the total energy transferred to the gas;

- the $\mathrm{H}_{2} \mathrm{O}$ effect is associated to the higher concentration of targets for the production of the dominant species $\mathrm{H}$ and $\mathrm{OH}_{i}$ furthermore, the water vapour increases the total energy transferred to the gas and makes possible to work at higher voltages lbecause of the higher guiding fieldl;

- it may be worth to mix in the flue gas some air and water vapour to increase the content of $\mathrm{O}_{2}$ and $\mathrm{H}_{2} \mathrm{O}$, before the corona induced DeNO $\mathrm{O}_{x}$ and DeSO $\mathrm{x}_{x}$ treatment: in this case it is possible to obtain $G$ values around $3+4$ radicals $/ 100 \mathrm{eV}$, very similar to those obtained with E-bean irradiation.

\section{CONCLUSIONS}

The model of impulse corona presented in this paper makes possible to follow the development of single streamer channels in space and time; macroscopic characteristics, as current pulse, net charge injection, energy losses, space charge distribution, radical densities, etc. can be derived, for any gas mixture, geometric configuration and applied voltage. The model can be therefore utilized as a useful tool to control the discharge conditions in practical applications for flue gas treatment.

The presented results put in evidence that the discharge behaviour is mainly controlled by the water vapour and oxygen concentrations: the $\mathrm{H}_{2} \mathrm{O}$ content defines the attachment rate in the ionization front and therefore controls the advancement conditions of the streamer head (guiding field, charge formation energy, streamer length etc.); the $\mathrm{O}_{2}$ content defines the three-body attachment in the streamer channel and therefore controls the electron current flow Inet charge, charge separation energy, total energy, radical production efficiency, etc. 1 .

To test the reliability of the model a prototipe cell is beeing installed in a power plant in order to collect experimental results in flue gases, under real operating conditions. The preliminary measurements present a general trend which is quite consistent with the model predictions.

\section{Acknowledgements}

The presented work has been carried on within the frame of the European Group on Gas Discharges (with the support of EEC); intense collaboration has been established with ENELC.R.T.N. - New Techniques Dept. (Pisa) and CNR-F.R.A.E. (Bologna),

The author is particularly indebt with Dr. S.Stangherlin and Dr. M. Bernardi for the valuable help in the development of the work and the preparation of the paper.

\section{REFERENCES}

1. G.Bondani, G.Dinelli, I.Gallimberti and M.Rea, IEEE - Proc. IAS85 Ann.Meet., 1285-1291, Toronto $(1985)$

2. G.Bondani, G.Dinel1i, I.Gallimberti and M.Rea, AEI Ann.Meet., 3.2.2, Firenze (1986)

3. L.Civitano, G.Dinelli, F.Busi, M.Dangelantonio, I.Gallimberti and M.Rea, IAEA Consult. Meet., Karlsruhe (1986)

4. I.Galiimberti, 5th Int.Symp. On Gaseous Dielectrics, Knoxville (1987)

5. L.B.Loeb, Electrical Coronas, Univ. of California Press, Berkeley (1965)

6. I.Gallimberti, J. de Physique, 40 C7, 193-250 (1979)

7. R.F.Grifeiths and C.T.Phelps, Quart.J.R.Mat.Soc. $102,419-426(1976)$

8. I.Gallimberti, J.Phys.DiApp1.Phys 5, 2179-2189(1972)

9. I.Gallimberti and N.Wiegart, J.Phys.D:Appl.Phys.12, 2351-2379(1986)

10. I.Gallimberti, 6 th Symp. on Transfer and Utilization of Particulate Control Technology EPRI - New orleans (1985)

11. E.E.Kunhardt, J.WU and B.Penetrante, POLY-WRI 1509-87, Polytechnic Univ. New York (1987)

12. M.Yousfi, Ph.D.'Thesis, Univ. P. Sabatier Toulouse (1986)

13. M.Mitchner and C.H.Kruger, Partially Ionized Gases, Willey, New York (1973)

14. P.Laborie, J.M.Rocard and J.A.Rees, Sections efficaces electroniques et coefficient macroscopiques, Dunod, Faris 11971 )

15. J.W.Gallagher and E.C.Beaty, JILA Inf.C.Rep. 18 and 21 , Boulder 11981$)$

16. P.Segur, M.Yousfi, M.H.Kadri and M.C.Bordage, Transp.Th. and Stat.Phys. 15, 705-757 $(1986)$

17. A.V.Phelps and L.C.Pitchford, JILA Inf.C.Rep. 26, Boulder $\{1985\}$ A.V.Phelps, JILA Inf.C.Rep. 28, Boulder (1985)

18. M.Yousfi, I.Gallimberti, S.Stangherlin and P.Segur, to be published.

19. I.Gallimberti, M.Yousfi, S.Stangherlin, N.Azzi and P.Segur, 18th I.C.P.I.G., Swansea $(1987)$

20. I.Gallimberti and B.Gleijeses, A.Ist.Ven.Scienze, Lettere ed Arti, 136 (1977)

21. R.Morrow and L.E.Cran, J.Comp.Phys. $65,111(1985)$

22. M.Dalla Francesca, Ph.D.Thesis, Padova Univ., 11987

23. F.Busi, M.Dangelantonio, Q.G.Mulazzini, V.Raffaelli and O.Tubertini, Radiat. Phys.Chem. $25,47-55(1985)$ 